\title{
A NEOHOOKEAN MODEL OF PLATES
}

\section{Iwaniec, Tadeusz}

2021

Iwaniec , T , Onninen , J , Pankka, P \& Radice , T 2021 , ' A NEOHOOKEAN MODEL OF

PLATES ' , SIAM Journal on Mathematical Analysis , vol. 53 , no. 1 , pp. 509-529 . https://doi.org/10.1137/20M13293

http://hdl.handle.net/10138/328644

https://doi.org/10.1137/20M1329305

unspecified

publishedVersion

Downloaded from Helda, University of Helsinki institutional repository.

This is an electronic reprint of the original article.

This reprint may differ from the original in pagination and typographic detail.

Please cite the original version. 


\title{
A NEOHOOKEAN MODEL OF PLATES*
}

\author{
TADEUSZ IWANIEC ${ }^{\dagger}$, JANI ONNINEN $^{\ddagger}$, PEKKA PANKKA ${ }^{\S}$, AND TERESA RADICEף
}

\begin{abstract}
This article is about hyperelastic deformations of plates (planar domains) which minimize a neohookean-type energy. Particularly, we investigate a stored energy functional introduced by J. M. Ball [Proc. Roy. Soc. Edinb. Sect. A, 88 (1981), pp. 315-328]. The mappings under consideration are Sobolev homeomorphisms and their weak limits. They are monotone in the sense of C. B. Morrey. One major advantage of adopting monotone Sobolev mappings lies in the existence of the energy-minimal deformations. However, injectivity is inevitably lost, so an obvious question to ask is, what are the largest subsets of the reference configuration on which minimal deformations remain injective? The fact that such subsets have full measure should be compared with the notion of global invertibility, which deals with subsets of the deformed configuration instead. In this connection we present a Cantor-type construction to show that both the branch set and its image may have positive area. Another novelty of our approach lies in allowing the elastic deformations to be free along the boundary, known as frictionless problems.
\end{abstract}

Key words. neohookean materials, minimizers, monotone mappings, he principle of noninterpenetration of matter

AMS subject classifications. 73C50, 35J25, 26B99

DOI. $10.1137 / 20 \mathrm{M} 1329305$

1. Introduction. We study hyperelastic deformations of neohookean materials in planar domains called plates. These problems are motivated by recent remarkable relations between geometric function theory $[3,17,18,34]$ and the theory of nonlinear elasticity $[1,4,9]$. Both theories are governed by variational principles. Here we confine ourselves to deformations of bounded Lipschitz domains $\mathbb{X}, \mathbb{Y} \subset \mathbb{R}^{2} \simeq \mathbb{C}$ of finite connectivity. The general theory of hyperelasticity deals with Sobolev homeomorphisms $h: \mathbb{X} \stackrel{\text { onto }}{\longrightarrow} \mathbb{Y}$ of nonnegative Jacobian determinant, $J_{h} \stackrel{\text { def }}{=} \operatorname{det} D h \geqslant 0$, which minimize a given stored energy functional

$$
\mathcal{E}[h] \stackrel{\text { def }}{=} \int_{\mathbb{X}} E(|D h|, \operatorname{det} D h) \mathrm{d} x, \text { where } E: \mathbb{R}_{+} \times \mathbb{R}_{+} \rightarrow \mathbb{R} .
$$

The stored energy function $E: \mathbb{R}_{+} \times \mathbb{R}_{+} \rightarrow \mathbb{R}$ is determined by the elastic and mechanical properties of the material.

Here the $2 \times 2$-matrix $D h \in \mathbb{R}^{2 \times 2}$ is referred to as the deformation gradient, and $|D h|$ denotes its Hilbert-Schmidt norm. We are largely concerned with orientationpreserving homeomorphisms $h: \mathbb{X} \stackrel{\text { onto }}{\longrightarrow} \mathbb{Y}$ of the Sobolev class $\mathscr{W}^{1, p}(\mathbb{X}, \mathbb{C})$, denoted by

* Received by the editors April 3, 2020; accepted for publication (in revised form) September 14, 2020; published electronically January 25, 2021.

https://doi.org/10.1137/20M1329305

Funding: The work of the first author was supported by the NSF through grant DMS-1802107. The work of the second author was supported by the NSF through grant DMS-1700274. The work of the third author was supported by the Academy of Finland through project \#297258.

${ }^{\dagger}$ Department of Mathematics, Syracuse University, Syracuse, NY 13244 USA (tiwaniec@syr.edu).

${ }^{\ddagger}$ Department of Mathematics, Syracuse University, Syracuse, NY 13244 USA and Department of Mathematics and Statistics, P.O. Box 35 (MaD) FI-40014 University of Jyväskylä, Finland (jkonnine@syr.edu).

$\S$ Department of Mathematics and Statistics, P.O. Box 68 (Gustaf Hällströmin katu 2b), FI-00014 University of Helsinki, Finland (pekka.pankka@helsinki.fi).

ฯUniversitá degli Studi di Napoli "Federico II," Dipartimento di Matematica e Applicazioni "R. Caccioppoli" Via Cintia, 80126 Napoli, Italy (teresa.radice@unina.it). 
$\mathscr{H}^{p}(\mathbb{X}, \mathbb{Y})$, as well as their weak and strong limits. If $p \geqslant 2$, then every $h \in \mathscr{H}^{p}(\mathbb{X}, \mathbb{Y})$ extends continuously up to the boundary, still denoted by $h: \overline{\mathbb{X}} \rightarrow \overline{\mathbb{Y}}$; see [22].

The term neohookean refers to a stored energy function $E$ which increases to infinity when $J_{h}$ approaches zero. The neohookean materials have gained a lot of interest in mathematical models of nonlinear elasticity $[7,8,11,13,16,32,33,35]$. In particular, one minimizes functionals which are composed by the sum of the $\mathscr{L}^{2}$-norm of the deformation gradient and a nonlinear function of $J_{h}$; see [36]. A more general model example takes the form

$$
\mathrm{E}_{q}^{p}[h]=\int_{\mathbb{X}}\left[|D h|^{p}+\frac{1}{(\operatorname{det} D h)^{q}}\right] \mathrm{d} x, \quad p>1 \text { and } q>0 .
$$

Throughout this paper we tacitly assume that the class of admissible homeomorphisms is nonempty; that is, there is $h \in \mathscr{H}^{p}(\mathbb{X}, \mathbb{Y})$ such that $\mathrm{E}_{q}^{p}[h]<\infty$. In particular, $\mathbb{X}$ and $\mathbb{Y}$ are of the same topological type. As a first step toward understanding the existence problems we shall accept the weak limits of energy-minimizing sequences of homeomorphisms as legitimate deformations. Thus, we allow so-called weak interpenetration of matter; precisely, squeezing of the material can occur. This changes the nature of the minimization problem to the extent that the minimal energy (usually attained) can be strictly smaller than the infimum energy among homeomorphisms.

In a seminal work of Ball [5], injectivity properties were studied for pure displacement problems. That is, the admissible deformations are specified a priori on the entire boundary of the reference configuration $\mathbb{X}$. More specifically, choose and fix $\varphi \in \mathscr{H}^{p}(\mathbb{X}, \mathbb{Y}), p \geqslant 2$, and introduce the following class of admissible deformations:

$$
\mathcal{A}^{p} \stackrel{\text { def }}{=}\left\{h \in \mathscr{C}(\overline{\mathbb{X}}, \mathbb{C}) \cap \mathscr{W}^{1, p}(\mathbb{X}, \mathbb{C}): J(x, h)>0 \text { a.e., } h=\varphi \text { on } \partial \mathbb{X}\right\} .
$$

Ball [5] proved the following:

- If $p>2$ and $q>0$, then there exist an energy-minimal map $h_{\circ} \in \mathcal{A}^{p}$ such that

$$
\mathrm{E}_{q}^{p}\left[h_{\circ}\right]=\inf _{h \in \mathcal{A}^{p}} \mathrm{E}_{q}^{p}[h] .
$$

- For every $h \in \mathcal{A}^{p}$ with $p>2$, the following set has full measure:

$$
\mathbb{Y}_{h} \stackrel{\text { def }}{=}\left\{y \in h(\overline{\mathbb{X}}): h^{-1}(y) \text { is a single point }\right\} \subset \overline{\mathbb{Y}} .
$$

This result has been referred to as global invertibility for two reasons: because $\mathbb{Y}_{h}$ has full measure in $\overline{\mathbb{Y}}$ and because any minimizer $h_{\circ}$ on restriction to $h_{\circ}^{-1}\left(\mathbb{Y}_{h_{\circ}}\right)$ becomes injective. For further generalizations of the global invertibility result when $p \geqslant 2$, we refer the reader to [14].

Ciarlet and Nečas [10] studied mixed boundary value problems (the displacement is prescribed only on a portion of the boundary of the reference configuration $\mathbb{X}$ ). In their mixed problems, the pure displacement condition $h=\varphi$ on $\partial \mathbb{X}$ is replaced by

$$
\int_{\mathbb{X}} \operatorname{det} D h(x) \mathrm{d} x \leqslant|h(\mathbb{X})| .
$$

They showed that the minimizers of $\mathrm{E}_{q}^{p}, p>2$, subject to such class of deformations are globally invertible.

Usually, in geometric function theory, the boundary values of homeomorphisms $h$ are not given. For example, extremal Teichmüller quasiconformal mappings are not prescribed on the boundary; the boundary does not even exist for compact Riemann 
surfaces. In nonlinear elasticity, this is interpreted as saying that the elastic deformations are allowed to slip along the boundary, known as frictionless problems $[4,6,9,10]$.

Our goal is to enlarge the class of homeomorphisms (as little as possible) to ensure the existence of minimizers in that class. The right way is to adopt the monotone Sobolev mappings [25]. Indeed, that this class is a bare minimal enlargement of homeomorphisms follows from a Sobolev variant of the classical Young's approximation theorem. Its classical topological setting asserts that a continuous map between compact oriented topological 2-manifolds (such as plates and thin films) is monotone if and only if it is a uniform limit of homeomorphisms. Monotonicity, the concept of Morrey [30], simply means that for a continuous $h: \overline{\mathbb{X}} \rightarrow \overline{\mathbb{Y}}$, the preimage $h^{-1}(C)$ of a continuum (a connected set) $C \subset \overline{\mathbb{Y}}$ is a continuum in $\overline{\mathbb{X}}$. The above-mentioned Sobolev variant reads as follows.

TheOREM 1.1 (approximation by Sobolev homeomorphisms ([25])). Let $\mathbb{X}$ and $\mathbb{Y}$ be bounded Lipschitz planar domains. Suppose that $h: \overline{\mathbb{X}} \stackrel{\text { onto }}{\longrightarrow} \overline{\mathbb{Y}}$ is a monotone Sobolev mapping in $\mathscr{W}^{1, p}\left(\mathbb{X}, \mathbb{R}^{2}\right), 1<p<\infty$. Then $h$ can be approximated in norm topology of $\mathscr{W}^{1, p}\left(\mathbb{X}, \mathbb{R}^{2}\right.$ ) (and uniformly) by homeomorphisms $h_{j}: \mathbb{X} \stackrel{\text { onto }}{\longrightarrow} \mathbb{Y}$.

Let us introduce the notation $\mathscr{M}^{p}(\overline{\mathbb{X}}, \overline{\mathbb{Y}})$ for the class of orientation-preserving monotone mappings $h: \overline{\mathbb{X}} \stackrel{\text { onto }}{\longrightarrow} \overline{\mathbb{Y}}$ in $\mathscr{W}^{1, p}(\mathbb{X}, \mathbb{C})$. We say that a mapping $h$ is orientation preserving if $\operatorname{det} D h \geqslant 0$ a.e. Our first result guarantees the existence of a minimizer of neohookean energy among Sobolev monotone deformations.

TheOREM 1.2. Let $p \geqslant 2$ and $q>0$. Then there exists $h_{\circ} \in \mathscr{M}^{p}(\overline{\mathbb{X}}, \overline{\mathbb{Y}})$ such that

$$
\mathrm{E}_{q}^{p}\left[h_{\circ}\right]=\inf _{h \in \mathscr{M}^{p}(\overline{\mathbb{X}}, \overline{\mathbb{Y}})} \mathrm{E}_{q}^{p}[h] .
$$

All the evidence points to the following.

Conjecture 1.3. Every minimizer $h_{\circ} \in \mathscr{M}^{p}(\overline{\mathbb{X}}, \overline{\mathbb{Y}})$ in (1.4) is a homeomorphism.

For example, this conjecture is confirmed when $\mathbb{X}$ and $\mathbb{Y}$ are circular annuli; see [21]. Also, the conjecture is valid if $\mathbb{X}=\mathbb{Y}$, in which case it is relatively easy to see that the identity map minimizes the energy; see Example 3.1. However, it is not known whether the identity map minimizes the neohookean energy $\mathrm{E}_{q}^{p}$ when $p<2$. It is worth noting that this is not the case for the $p$-harmonic energy; see (3.2).

We give an affirmative answer to these questions for neohookean materials whose associated energy integrand grows sufficiently fast. Precisely, we have the following.

THEOREM 1.4. Let $p>2$ and $q>1$ such that $\frac{2}{p}+\frac{1}{q} \leqslant 1$. Then there exists a homeomorphism $h_{\circ} \in \mathscr{H}^{p}(\mathbb{X}, \mathbb{Y})$ such that

$$
\mathrm{E}_{q}^{p}\left[h_{\circ}\right]=\inf _{h \in \mathscr{M}^{p}(\overline{\mathbb{X}}, \overline{\mathbb{Y}})} \mathrm{E}_{q}^{p}[h]=\inf _{h \in \mathscr{H}^{p}(\mathbb{X}, \mathbb{Y})} \mathrm{E}_{q}^{p}[h] .
$$

The existence of monotone minimizer $h_{\circ}$ is ensured by Theorem 1.2, and the fact that $h_{\circ}$ is a homeomorphism follows from the next result.

TheOrem 1.5. Let $p>2$ and $q \geqslant \frac{p}{p-2}$. If $h \in \mathscr{M}^{p}(\overline{\mathbb{X}}, \overline{\mathbb{Y}})$ and

$$
\int_{\Omega}\left(|D h|^{p}+\frac{1}{J_{h}^{q}}\right) \mathrm{d} x<\infty \text { for every compact subset } \Omega \Subset \mathbb{X},
$$

then $h: \mathbb{X} \stackrel{\text { onto }}{\longrightarrow} \mathbb{Y}$ is a homeomorphism. 
Remark 1.6. Theorem 1.5 also holds for $p=2$ and $q=\infty$, in which case the locally finite energy condition (1.5) should be stated as

$$
\int_{\mathbb{X}}|D h|^{2} \mathrm{~d} x<\infty \text { and } \frac{1}{\operatorname{det} D h} \in \mathscr{L}^{\infty}(\mathbb{X}) .
$$

That is, $\operatorname{det} D h(x) \geqslant \frac{1}{C}>0$ with a constant $C=\left\|J_{h}^{-1}\right\|_{\mathscr{L} \infty(\mathbb{X})}$.

Theorem 1.5 is sharp; namely, it fails if $0<q<\frac{p}{p-2}$, as the following example shows.

Example 1.7. For $0<q<\frac{p}{p-2}$, there exists a noninjective $h \in \mathscr{M}^{p}(\overline{\mathbb{X}}, \overline{\mathbb{Y}})$ with $\mathrm{E}_{q}^{p}[h]<\infty$.

This example raises a question about partial injectivity of $h \in \mathscr{M}^{p}(\overline{\mathbb{X}}, \overline{\mathbb{Y}})$ with $\mathrm{E}_{q}^{p}[h]<\infty$ when $0<q<\frac{p}{p-2}$. First, we have the following.

TheOREM 1.8. Suppose that a monotone map $h: \overline{\mathbb{X}} \stackrel{\text { onto }}{\longrightarrow} \overline{\mathbb{Y}}$ of Sobolev class $\mathscr{W}^{1,2}(\mathbb{X}, \mathbb{C})$ has positive Jacobian determinant a.e. Then

- $h$ is globally invertible in the sense of (1.2);

- in addition, there exists $\mathbb{X}_{h}$ of full measure in $\mathbb{X}$ such that $h$ restricted to $\mathbb{X}_{h}$ is injective.

In particular, every minimizer $h_{\circ}$ in Theorem 1.2 is globally invertible in the sense of $(1.2)$.

Next in line is the study of the branch set

$$
\mathcal{B}_{h} \stackrel{\text { def }}{=}\{x \in \mathbb{X}: h \text { fails to be homeomorphic near } x\}
$$

and its image $h\left(\mathcal{B}_{h}\right)$. Recall that for the Dirichlet energy, the branch set of the energy-minimal mapping $h \in \mathscr{M}^{2}(\overline{\mathbb{X}}, \overline{\mathbb{Y}})$ may have a positive area, whereas $h\left(\mathcal{B}_{h}\right) \subset$ $\partial \mathbb{Y}$ (actually a nonconvex part of $\partial \mathbb{Y}$ ) [12, 23]. We show, however, that under the assumptions of Example 1.7, both the branch set and also the image of the branch set may have a positive area. Recall that if $q \geqslant \frac{p}{p-2}>0$, then any monotone map $h$ with $\mathrm{E}_{q}^{p}[h]<\infty$ is injective by Theorem 1.5.

Example 1.9. If $0<q<\frac{p}{p-2}$, then there exists $h \in \mathscr{M}^{p}(\overline{\mathbb{X}}, \overline{\mathbb{Y}})$ with $\mathrm{E}_{q}^{p}[h]<\infty$ such that $\left|\mathcal{B}_{h}\right|>0$ and $\left|h\left(\mathcal{B}_{h}\right)\right|>0$.

Our example is based on a Cantor-type construction; see section 6 for the construction.

Returning to Conjecture 1.3, with the aid of the complex partial derivatives, $h_{z}=\frac{\partial h}{\partial z}$ and $h_{\bar{z}}=\frac{\partial h}{\partial \bar{z}}$, we express the energy as

$$
\mathrm{E}_{1}^{2}[h]=\int_{\mathbb{X}}\left[2\left(\left|h_{z}\right|^{2}+\left|h_{\bar{z}}\right|^{2}\right)+\left(\left|h_{z}\right|^{2}-\left|h_{\bar{z}}\right|^{2}\right)^{-1}\right] \mathrm{d} z .
$$

Clearly, one cannot perform outer variations $h_{\varepsilon}=h+\varepsilon \eta$, with $\eta \in \mathscr{C}_{\circ}^{\infty}(\mathbb{X})$, as they live out the class of monotone Sobolev mappings $\mathscr{M}^{p}(\overline{\mathbb{X}}, \overline{\mathbb{Y}})$. Thus, we lose the EulerLagrange equation, which is the major source of difficulty here. Such a difficulty is widely recognized in the theory of nonlinear elasticity. This forces us to rely on the inner variation of the independent variable $z_{\varepsilon}=z+\varepsilon \tau(z)$, where $\tau \in \mathscr{C}_{\circ}^{\infty}\left(\mathbb{X}, \mathbb{R}^{2}\right)$. 
The inner variational equation for the minimizer takes the form

$$
\frac{\partial}{\partial z}\left[\left(1-\frac{p}{2}\right)|D h|^{p}+\frac{1+q}{J_{h}^{q}}\right]=2 p \frac{\partial}{\partial \bar{z}}\left[|D h|^{p-2} \overline{h_{z}} h_{\bar{z}}\right] ;
$$

see formula (2.6), page 648 in [20].

Here, the complex partial derivatives $\frac{\partial}{\partial z}$ and $\frac{\partial}{\partial \bar{z}}$ are understood in the sense of distributions. For $p=2$ and $q=1$, this simplifies as

$$
2 \frac{\partial}{\partial \bar{z}}\left[h_{z} \overline{h_{\bar{z}}}\right]=\frac{\partial}{\partial z}\left[\frac{1}{\operatorname{det} D h}\right], \quad \text { with } \mathrm{E}_{1}^{2}[h]<\infty .
$$

Let us name (1.7) and (1.8) neohookean Hopf systems.

It is worth noting that monotone Lipschitz solutions to the neohookean Hopf system (1.8) are homeomorphisms. In this connection we recall that for the Dirichlet energy, the inner-stationary solutions are always Lipschitz continuous; see [20]. Actually, a solution of (1.8) in $\mathscr{M}^{4}(\overline{\mathbb{X}}, \overline{\mathbb{Y}})$ will turn out to be a homeomorphism. This follows from the next result, simply by taking $p=2$ and $q=1$.

TheOrEm 1.10. Consider a monotone mapping $h: \overline{\mathbb{X}} \stackrel{\text { onto }}{\longrightarrow} \overline{\mathbb{Y}}$ of finite neohookean energy:

$$
\mathrm{E}_{q}^{p}[h]=\int_{\mathbb{X}}\left(|D h|^{p}+J_{h}^{-q}\right) \mathrm{d} x, \quad p>1 \quad \text { and } \quad q>0 .
$$

Assume that $h \in \mathscr{W}_{\text {loc }}^{1, s}\left(\mathbb{X}, \mathbb{R}^{2}\right)$ for some $s \geqslant \frac{p}{q}+2$ and $s>p$ satisfies the equation (1.7). Then $h$ is a homeomorphism of $\mathbb{X}$ onto $\mathbb{Y}$.

\section{Preliminaries.}

2.1. Monotone in the sense of Lebesgue. There are several notions commonly known in literature as monotonicity. To avoid confusion we use the term monotone in the sense of Lebesgue for one of these. This notion goes back to Lebesgue [29] in 1907.

Definition 2.1. Let $\mathbb{X}$ be an open subset of $\mathbb{R}^{2}$. A continuous mapping $h: \mathbb{X} \rightarrow$ $\mathbb{R}^{2}$ is monotone in the sense of Lebesgue if for every open set $\Omega \subset \mathbb{X}$ we have

$$
\operatorname{diam} h(\bar{\Omega})=\operatorname{diam} h(\partial \Omega) .
$$

Note that for a real-valued function, (2.1) can be stated as

$$
\begin{aligned}
& \min _{\bar{\Omega}} h=\min _{\partial \Omega} h \text { (minimum principle) } \\
& \max _{\bar{\Omega}} h=\max _{\partial \Omega} h \quad \text { (maximum principle). }
\end{aligned}
$$

2.2. Modulus of continuity and conformal energy. In the next lemma, $\mathbb{X}$ and $\mathbb{Y}$ are $\ell$-connected Lipschitz domains in $\mathbb{R}^{2}$; see [26, Lemma 4.3].

Lemma 2.2. To every pair $(\mathbb{X}, \mathbb{Y})$ of $\ell$-connected bounded Lipschitz domains, $\ell \geqslant$ 2 , there corresponds a constant $C=C(\mathbb{X}, \mathbb{Y})$ such that for $h \in \mathscr{H}^{2}(\mathbb{X}, \mathbb{Y})$ and $\ell \geqslant 2$ we have

$$
\left|h\left(x_{1}\right)-h\left(x_{2}\right)\right|^{2} \leqslant \frac{C \cdot \int_{\mathbb{X}}|D h|^{2}}{\log \left(1+\frac{\operatorname{diam} \mathbb{X}}{\left|x_{1}-x_{2}\right|}\right)}, \quad x_{1}, x_{2} \in \overline{\mathbb{X}},
$$

whenever $h \in \mathscr{H}^{2}(\mathbb{X}, \mathbb{Y})$ and $x_{1} \neq x_{2}$ in $\overline{\mathbb{X}}$.

Copyright (c) by SIAM. Unauthorized reproduction of this article is prohibited. 
Remark 2.3. Inequality (2.4) fails when $\ell=1$ and $p=2$. For this, consider a sequence of the Möbius transformations $h_{k}: \mathbb{D} \stackrel{\text { onto }}{\longrightarrow} \mathbb{D}, k=1,2, \ldots$

$$
h_{k}(z)=\frac{z+a_{k}}{1+a_{k} z}, \quad 0<a_{k}<1 \quad \text { and } a_{k} \nearrow 0 .
$$

The mappings are fixed at two boundary points,

$$
h_{k}(1)=1 \quad \text { and } \quad h_{k}(-1)=-1,
$$

and are equiintegrable:

$$
\int_{\mathbb{X}}\left|D h_{k}\right|=2 \int_{\mathbb{X}} J_{h}(x) \mathrm{d} x=2 \pi .
$$

The sequence $h_{k}: \mathbb{D} \stackrel{\text { onto }}{\longrightarrow} \mathbb{D}$ approaches the constant mapping $h(z) \equiv 1$. Obviously, we are losing equicontinuity of the boundary mappings $h_{k}: \partial \mathbb{D} \stackrel{\text { onto }}{\longrightarrow} \partial \mathbb{D}$, in contradiction with (2.4).

2.3. Change of variables formula. We say that $h: \mathbb{X} \rightarrow \mathbb{C}$ satisfies the Lusin (N) condition if for every $E \subset \mathbb{X}$ such that $|E|=0$ we have $|h(E)|=0$.

Lemma 2.4. Suppose that $h \in \mathscr{W}^{1,2}(\mathbb{X}, \mathbb{C})$ with $J_{h}>0$ a.e. Then $h$ satisfies the Lusin (N) condition.

Lemma 2.4 follows because a monotone mapping in the sense of Lebesgue in the Sobolev class $\mathscr{W}_{\text {loc }}^{1,2}(\mathbb{X}, \mathbb{C})$ satisfies the Lusin $(\mathrm{N})$ condition; see, e.g., [15, Lemma 1.2]. On the other hand, a mapping $h \in \mathscr{W}^{1,2}(\mathbb{X}, \mathbb{C})$ with $J_{h}>0$ a.e. is monotone in the sense of Lebesgue; see [19, Proposition 4.1]. The Lusin property is very important, as it allows us to obtain the change of variables formula; see [18, Theorem 6.3.2].

Lemma 2.5. Let $h: \mathbb{X} \rightarrow \mathbb{C}$ be a mapping in the Sobolev class $\mathscr{W}^{1,2}(\mathbb{X}, \mathbb{C})$ with $J_{h}(x)>0$ for almost every $x$ in $\mathbb{X}$. If $\eta$ is a nonnegative Borel measurable function on $\mathbb{C}$ and $A$ a Borel measurable set in $\mathbb{X}$, then

$$
\int_{A} \eta(h(x)) J_{h}(x) \mathrm{d} x=\int_{h(A)} \eta(y) N_{h}(y, A) \mathrm{d} y,
$$

where $N_{h}(y, A)$ denotes the cardinality of the set $\{x \in A: h(x)=y\}$.

\subsection{Weak compactness of Jacobians.}

Lemma 2.6. Let $\mathbb{X}$ be a domain in $\mathbb{C}$ and $h_{k} \in \mathscr{W}^{1,2}(\mathbb{X}, \mathbb{C})$ a sequence of mappings with $J\left(x, h_{k}\right) \geqslant 0$ a.e. in $\mathbb{X}$ converging weakly in $\mathscr{W}^{1,2}(\mathbb{X}, \mathbb{C})$ to $h \in \mathscr{W}^{1,2}(\mathbb{X}, \mathbb{C})$. Then the Jacobians $J\left(x, h_{k}\right)$ converge weakly in $\mathscr{L}_{\mathrm{loc}}^{1}(\mathbb{X})$ to $J(x, h)$ and $J(x, h) \geqslant 0$ a.e. in $\mathbb{X}$. Precisely,

$$
\lim _{k \rightarrow \infty} \int_{\mathbb{X}} \varphi(x) J\left(x, h_{k}\right) \mathrm{d} x=\int_{\mathbb{X}} \varphi(x) J(x, h) \mathrm{d} x
$$

for every $\varphi \in \mathscr{L}^{\infty}(\mathbb{X})$ with compact support.

For a proof of this lemma we refer the reader to [18, Theorem 8.4.2].

2.5. Polyconvexity of neohookean integrand. The remarkable feature of the neohookean energy is the polyconvexity of its integrand. Instead of the general definition $[4,31]$, let us confine ourselves, as a consequence, to the so-called gradient inequalities. 
Let $p \geqslant 2$ and $q>0$. For arbitrary square matrices $A \in \mathbb{R}^{2 \times 2}$ and $A_{\circ} \in \mathbb{R}^{2 \times 2}$, we have

$$
\begin{aligned}
|A|^{p}-\left|A_{\circ}\right|^{p} & =\left(|A|^{2}\right)^{\frac{p}{2}}-\left(\left|A_{\circ}\right|^{2}\right)^{\frac{p}{2}} \geqslant \frac{p}{2}\left(\left|A_{\circ}\right|^{2}\right)^{\frac{p}{2}-1}\left(|A|^{2}-\left|A_{\circ}\right|^{2}\right) \\
& \geqslant \frac{p}{2}\left|A_{\circ}\right|^{p-2} 2\left\langle A_{\circ}, A-A_{\circ}\right\rangle=\left\langle p\left|A_{\circ}\right|^{p-2} A_{\circ}, A-A_{\circ}\right\rangle,
\end{aligned}
$$

where $\langle\cdot, \cdot\rangle$ stands for the scalar product of matrices.

For arbitrary positive numbers $J$ and $J_{0}$, we have

$$
\frac{1}{J^{q}}-\frac{1}{J_{\circ}^{q}} \geqslant \frac{q}{J_{\circ}^{q+1}}\left(J_{\circ}-J\right)
$$

Next we show that the lower semicontinuity of neohookean integral follows from the above gradient inequalities.

Lemma 2.7. Let $\mathbb{X}$ be a domain in $\mathbb{C}, p \geqslant 2$ and $q>0$. Suppose that $h_{k} \in$ $\mathscr{W}^{1, p}(\mathbb{X}, \mathbb{C})$ is a sequence of mappings with $J\left(x, h_{k}\right)>0$ a.e. in $\mathbb{X}$ converging weakly in $\mathscr{W}^{1, p}(\mathbb{X}, \mathbb{C})$ to $h \in \mathscr{W}^{1, p}(\mathbb{X}, \mathbb{C})$ and $\mathrm{E}_{q}^{p}\left[h_{k}\right] \leqslant E<\infty$. Then

$$
\mathrm{E}_{q}^{p}[h] \leqslant \liminf _{k \rightarrow \infty} \mathrm{E}_{q}^{p}\left[h_{k}\right] .
$$

Proof. Choose and fix a positive number $\varepsilon$ and a compact subset $F \Subset \mathbb{X}$. The above gradient inequalities imply

$$
\begin{aligned}
\int_{F} & {\left[\left|D h_{k}(x)\right|^{p}+\left[\varepsilon+J\left(x, h_{k}\right)\right]^{-q}\right]-\int_{F}\left[|D h(x)|^{p}+[\varepsilon+J(x, h)]^{-q}\right] } \\
\geqslant & p \int_{F}\left\langle|D h(x)|^{p-2} D h(x), D h_{k}(x)-D h(x)\right\rangle \mathrm{d} x \\
& +q \int_{F} \frac{J(x, h)-J\left(x, h_{k}\right)}{[\varepsilon+J(x, h)]^{q+1}} \mathrm{~d} x .
\end{aligned}
$$

Now letting $k \rightarrow \infty$, the first integral term goes to zero because $D h_{k}-D h \rightarrow 0$ weakly in $\mathscr{L}^{p}(F)$, whereas $|D h|^{p-2} D h$ belongs to the dual space of $\mathscr{L}^{p}(F)$. Concerning the last integral term, we appeal to Lemma 2.6 on weak compactness of the Jacobian determinants. Accordingly,

$$
\int_{F} \frac{J(x, h)-J\left(x, h_{k}\right)}{[\varepsilon+J(x, h)]^{q+1}} \mathrm{~d} x \rightarrow 0,
$$

where our test function $\varphi(x)=\frac{\chi_{F}(x)}{[\varepsilon+J(x, h)]^{q+1}} \leqslant \frac{1}{\varepsilon}$ lies in $\mathscr{L}^{\infty}(\mathbb{X})$ and has compact support. We thus have an estimate

$$
\begin{aligned}
& \int_{F}\left[|D h(x)|^{p}+[\varepsilon+J(x, h)]^{-q}\right] \mathrm{d} x \\
& \leqslant \liminf _{k \rightarrow \infty} \int_{F}\left[\left|D h_{k}(x)\right|^{p}+\left[\varepsilon+J\left(x, h_{k}\right)\right]^{-q}\right] \mathrm{d} x \\
& \leqslant \liminf _{k \rightarrow \infty} \int_{\mathbb{X}}\left[\left|D h_{k}(x)\right|^{p}+\left[J\left(x, h_{k}\right)\right]^{-q}\right] \mathrm{d} x=\liminf _{k \rightarrow \infty} \mathrm{E}_{q}^{p}\left[h_{k}\right]<\infty .
\end{aligned}
$$

Consider a sequence $\left\{\varepsilon_{j}\right\}$ of positive numbers converging to zero and an increasing sequence of compact subsets $F_{1} \subset F_{2} \subset \ldots$ with $\bigcup F_{n}=\mathbb{X}$. Thus,

$$
\int_{F_{n}}\left[|D h(x)|^{p}+\left[\varepsilon_{n}+J(x, h)\right]^{-q}\right] \mathrm{d} x \leqslant \liminf _{k \rightarrow \infty} \mathrm{E}_{q}^{p}\left[h_{k}\right]<\infty .
$$

Copyright $\odot$ by SIAM. Unauthorized reproduction of this article is prohibited. 
Letting $n \rightarrow \infty$, by the monotone convergence theorem, the desired estimate $\mathrm{E}_{q}^{p}[h] \leqslant$ $\liminf \inf _{k \rightarrow \infty} \mathrm{E}_{q}^{p}\left[h_{k}\right]$ follows.

3. The case of $\mathbb{X}=\mathbb{Y}$. When $\mathbb{X}=\mathbb{Y}$, the identity map is a natural candidate for the minimizer. The case $1<p<2$, however, offers further challenges. First of all, when $p \geqslant 2$ we have the following.

Example 3.1. The identity mapping $h_{\circ}=\mathrm{Id}: \mathbb{X} \stackrel{\text { onto }}{\longrightarrow} \mathbb{X}$ minimizes the neohookean energy $\mathrm{E}_{q}^{p}$ when $p \geqslant 2$ and $q>0$ subject to all homeomorphisms in $\mathscr{H}^{p}(\mathbb{X}, \mathbb{X})$. In fact, this follows from the inequality

$$
\mathrm{E}_{q}^{p}[h] \geqslant\left(2^{\frac{p}{2}}+1\right)|\mathbb{X}|=\mathrm{E}_{q}^{p}\left[h_{\circ}\right] \quad \text { for all } h \in \mathscr{H}^{p}(\mathbb{X}, \mathbb{X}) .
$$

The proof of this inequality is obtained by the methods of free Lagrangians. A free Lagrangian is a special case of null Lagrangian [4]. This is a nonlinear differential 2 -form defined on Sobolev homeomorphisms $h: \mathbb{X} \stackrel{\text { onto }}{\longrightarrow} \mathbb{Y}$ whose integral depends only on the homotopy class of $h$; see [23]. The simplest free Lagrangians is the area form $\operatorname{det} D h(x) \mathrm{d} x$ for $h \in \mathscr{W}^{1, p}(\mathbb{X}, \mathbb{X})$ with $p \geqslant 2$. This is a key player in the proof of (3.1). The unavailability of the area form is exactly why our arguments for Theorem 1.5 do not apply when $p<2$. Nevertheless, it is not clear whether (3.1) remains valid for $p<2$. In [28] it is shown that the identity mapping $h^{*}=\mathrm{Id}: \mathbb{D}_{\circ} \stackrel{\text { onto }}{\longrightarrow} \mathbb{D}_{\circ}$ from the punctured disk $\mathbb{D}_{\circ}=\{z \in \mathbb{C}: 0<|z|<1\}$ onto itself does not minimize the $p$-harmonic energy when $1 \leqslant p<p_{1}$ for some $1<p_{1}<2$. Namely,

$$
\inf _{h \in \mathscr{H}^{p}\left(\mathbb{D}_{\circ}, \mathbb{D}_{\circ}\right)} \int_{\mathbb{A}}|D h(x)|^{p} \mathrm{~d} x<\int_{\mathbb{A}}\left|D h^{*}(x)\right|^{p} \mathrm{~d} x .
$$

Let us point out that the identity mapping is always a minimizer in the class of radially symmetric homeomorphisms.

Proof of Example 3.1. First, applying Young's inequality $a b \leqslant \frac{a^{\alpha}}{\alpha}+\frac{b^{\beta}}{\beta}, \frac{1}{\alpha}+\frac{1}{\beta}=1$, we observe a pointwise inequality

$$
|D h|^{p} \geqslant p 2^{\frac{p-4}{2}}|D h|^{2}-(p-2) 2^{\frac{p-2}{2}} .
$$

Equality occurs if $|D h|^{2}=2$. Then Hadamard's inequality $|D h|^{2} \geqslant 2 J_{h}, J_{h}=\operatorname{det} D h$, yields

$$
|D h|^{p} \geqslant p 2^{\frac{p-2}{2}}\left[J_{h}-1+\frac{2}{p}\right] .
$$

Again, we have the equality when $h=$ Id. Hence,

$$
|D h|^{p}+\frac{1}{J_{h}^{q}} \geqslant\left(p 2^{\frac{p-2}{2}}-q\right) J_{h}-(p-2) 2^{\frac{p-2}{2}}+q J_{h}+\frac{1}{J_{h}^{q}},
$$

where $q J_{h}+\frac{1}{J_{h}^{q}} \geqslant q+1$. Therefore,

$$
|D h|^{p}+\frac{1}{J_{h}^{q}} \geqslant\left(p 2^{\frac{p-2}{2}}-q\right) J_{h}-(p-2) 2^{\frac{p-2}{2}}+q+1 .
$$

This gives us a desired estimate of the stored energy integrand by means of free Lagrangians, namely, $J_{h}$ and a constant function. Integrating over the domain $\mathbb{X}=\mathbb{Y}$, the claimed estimate (3.1) follows. Equality occurs for the identity map and only for isometries $h: \mathbb{X} \stackrel{\text { onto }}{\longrightarrow} \mathbb{X}$. 


\section{Proof of Theorem 1.8 .}

Proof. Step 1. $\left(\left|\mathbb{Y}_{\boldsymbol{h}}\right|=|\mathbb{Y}|\right)$. First, since $h \in \mathscr{W}^{1,2}(\mathbb{X}, \mathbb{C})$ and $J_{h}(x)>0$ a.e., Lemma 2.5 gives

$$
\int_{\mathbb{X}} J_{h}(x) \mathrm{d} x=\int_{\mathbb{Y}} N_{h}(y, \mathbb{X}) \mathrm{d} y
$$

where $N_{h}(y, \mathbb{X})$ denotes the cardinality of the set $\{x \in \mathbb{X}: h(x)=y\}$.

Second, for an orientation-preserving homeomorphism $g: \mathbb{X} \stackrel{\text { onto }}{\longrightarrow} \mathbb{Y}$ in the Sobolev class $\mathscr{W}^{1,2}(\mathbb{X}, \mathbb{C})$, we have

$$
\int_{\mathbb{X}} J_{g}(x) \mathrm{d} x=|\mathbb{Y}|
$$

Now combining this with Theorem 1.1 and Lemma 2.6 for an orientation-preserving monotone $h: \mathbb{X} \stackrel{\text { onto }}{\longrightarrow} \mathbb{Y}$ in $\mathscr{W}^{1,2}(\mathbb{X}, \mathbb{C})$, we have

$$
\int_{\mathbb{X}} J_{h}(x) \mathrm{d} x=|\mathbb{Y}|
$$

Therefore, by (4.1) and (4.3) for a monotone $h: \mathbb{X} \stackrel{\text { onto }}{\longrightarrow} \mathbb{Y}$ in $\mathscr{W}^{1,2}(\mathbb{X}, \mathbb{C})$ with $J_{h}(x)>0$ a.e. in $\mathbb{X}$, we obtain $N_{h}(y, \mathbb{X})=1$ for a.e. $y$ in $\mathbb{Y}$; that is, $\left|\mathbb{Y}_{h}\right|=|\mathbb{Y}|$. Since $\mathbb{Y}$ is a Lipschitz domain, it holds that $|\partial \mathbb{Y}|=0$.

Step 2. $\left(\left|\mathbb{X}_{\boldsymbol{h}}\right|=|\mathbb{X}|\right)$. The claim is that $\left|h^{-1}\left(\mathbb{Y}_{h}\right)\right|=|\mathbb{X}|$. Indeed, according to Lemma 2.5, we have

$$
\int_{\mathbb{X} \backslash h^{-1}\left(\mathbb{Y}_{h}\right)} J_{h}(x)=\left|\mathbb{Y} \backslash \mathbb{Y}_{h}\right|=0 .
$$

Furthermore, since $J_{h}(x)>0$ a.e. in $\mathbb{X}$, we have $\left|\mathbb{X} \backslash h^{-1}\left(\mathbb{Y}_{h}\right)\right|=0$.

\section{Constructing Example 1.7.}

Proof of Example 1.7. Consider the rectangles $\mathbb{X}=(-1,1) \times(-2,2)=\mathbb{Y}$. To construct a monotone map $h: \overline{\mathbb{X}} \stackrel{\text { onto }}{\longrightarrow} \overline{\mathbb{Y}}$, we choose and fix parameters $a>-\frac{1}{p}$; $b>1-\frac{1}{p}$ such that $a+b<\frac{1}{q}$. This choice is possible because $0<q<\frac{p}{p-2}$. The map in question is defined by the rule

$$
\begin{gathered}
h(x, y)=(u(x, y), v(x, y)), \text { where } \\
u(x, y)=x|x|^{a} \text { for }-1 \leqslant x \leqslant 1, \\
v(x, y)=\left\{\begin{array}{l}
y|x|^{b} \text { for }|y| \leqslant 1, \\
\left(2-|x|^{b}\right) y+2\left(|x|^{b}-1\right) \frac{y}{|y|} \text { for } 1 \leqslant|y| \leqslant 2 .
\end{array}\right.
\end{gathered}
$$

It is worth noting that for $x$ fixed the function $y \rightarrow v(x, y)$ is linear in each of the following intervals: $y \in[-1,1], y \in[1,2]$, and $y \in[-2,-1]$; see Figure 1. Clearly, we have

$$
h^{-1}(0,0)=\mathbb{I} \stackrel{\text { def }}{=}\{(0, y):|y| \leqslant 1\} .
$$

Outside this interval, $h$ is a bijection $h: \overline{\mathbb{X}} \backslash \mathbb{I} \stackrel{\text { onto }}{\longrightarrow} \overline{\mathbb{Y}} \backslash\{(0,0)\}$. Its inverse $h^{-1}: \overline{\mathbb{Y}} \backslash$ 


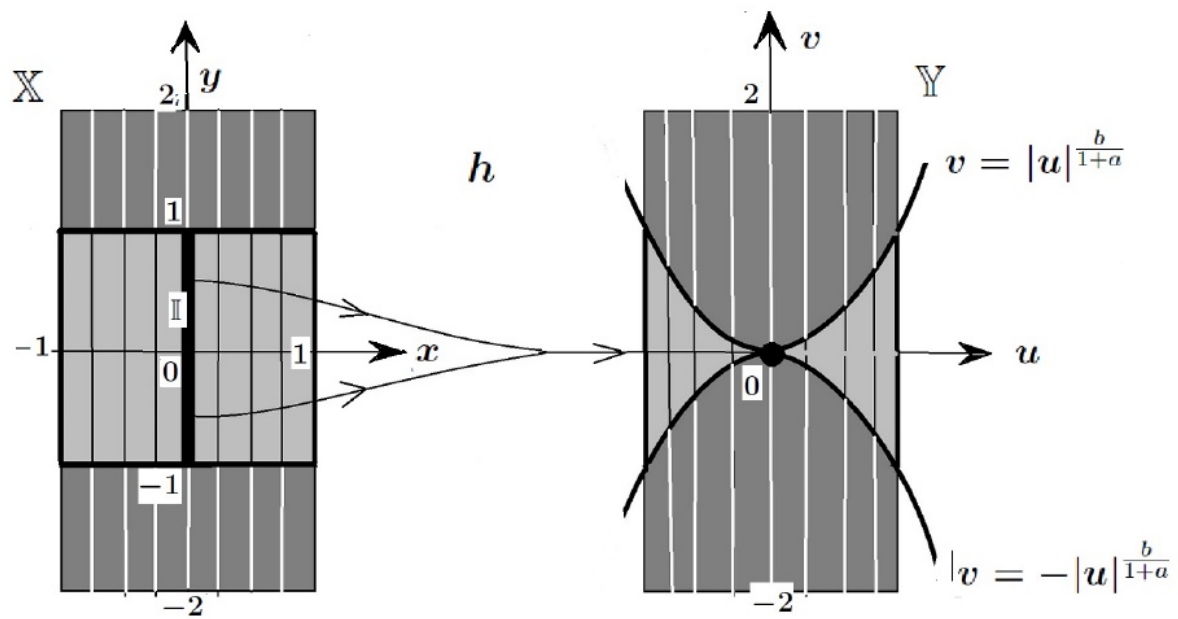

FIG. 1.

$\{(0,0)\} \stackrel{\text { onto }}{\longrightarrow} \overline{\mathbb{X}} \backslash \mathbb{I}$ takes the form $f(u, v)=(x(u, v), y(u, v))$, where

$$
\begin{gathered}
\qquad x(u, v)=u|u|^{\frac{-a}{1+a}},-1 \leqslant u \leqslant 1 \\
y(u, v)= \begin{cases}\frac{v \pm 2\left(1-|u|^{\frac{b}{1+a}}\right)}{2-|u|^{\frac{b}{1+a}}} & \text { whenever } \pm v \geqslant|u|^{\frac{b}{1+a}}, \text { respectively, } \\
v|u|^{\frac{-b}{1+a}} & \text { whenever }|v| \leqslant|u|^{\frac{b}{1+a}}\end{cases}
\end{gathered}
$$

Thereby, $h$ is a monotone map because $h^{-1}(0,0)=\mathbb{I}$ and outside this interval $h$ is an injective.

Concerning the energy of $h$, because of symmetries it is enough to evaluate the energy over the rectangle $(0,1) \times(0,2)$. The formula takes the form

$$
\mathrm{E}_{q}^{p}[h]=4 \int_{0}^{1}\left[\int_{0}^{1} \mathrm{E}(x, y) \mathrm{d} y+\int_{1}^{2} \mathrm{E}(x, y) \mathrm{d} y\right] \mathrm{d} x,
$$

where

$$
\mathrm{E}(x, y)=|D h(x, y)|^{p}+\frac{1}{J_{h}(x, y)^{q}} \text { for } x, y \geqslant 0 .
$$

Consider two cases:

Case $1.0 \leqslant y \leqslant 1$, so

$$
\begin{cases}u(x, y)=x^{a+1}, & \text { so } u_{x}=(a+1) x^{a} \text { and } u_{y}=0 \\ v(x, y)=x^{b} y, & \text { so } v_{x}=b x^{b-1} y \text { and } v_{y}=x^{b}\end{cases}
$$

Hence, $|D h(x, y)|^{p} \leqslant C x^{a p}+x^{(b-1) p}$ and $J_{h}(x, y)=u_{x} v_{y}-u_{y} v_{x}=u_{x} v_{y}=(a+1) x^{a+b}$. Since $a p>-1$ and $(b-1) p>-1$, we have

$$
\int_{0}^{1} \int_{0}^{1}|D h(x, y)|^{p} \mathrm{~d} x \mathrm{~d} y<\infty .
$$


On the other hand, $J_{h}(x, y)^{q}=(a+1)^{q} x^{(a+b) q}$. Since $a+b<\frac{1}{q}$, we have

$$
\int_{0}^{1} \int_{0}^{1} \frac{\mathrm{d} x \mathrm{~d} y}{J_{h}(x, y)^{q}}<\infty .
$$

In conclusion, the energy over the square $0 \leqslant x \leqslant 1,0 \leqslant y \leqslant 1$ is finite.

Case $2.1 \leqslant y \leqslant 2$, so

$$
\begin{cases}u(x, y)=x^{a+1}, & \text { so } u_{x}=(a+1) x^{a} \text { and } u_{y}=0 \\ v(x, y)=2 y-x^{b} y+2 x^{b}-2, & \text { so } v_{x}=b x^{b-1}(2-y) \text { and } v_{y}=2-x^{b} .\end{cases}
$$

Hence,

$$
\begin{gathered}
|D h(x, y)|^{p} \leqslant C x^{a p}+x^{(b-1) p} \\
J_{h}(x, y)=u_{x} v_{y}-u_{y} v_{x}=u_{x} v_{y}=(a+1) x^{a}\left(2-x^{b}\right) \geqslant(a+1) x^{a} .
\end{gathered}
$$

As in Case 1,

$$
\int_{0}^{1} \int_{0}^{1}|D h(x, y)|^{p} \mathrm{~d} x \mathrm{~d} y<\infty
$$

On the other hand,

$$
\int_{0}^{1} \int_{0}^{1} \frac{\mathrm{d} x \mathrm{~d} y}{J_{h}(x, y)^{q}} \leqslant \frac{1}{(a+1)^{q}} \int_{0}^{1} \int_{0}^{1} \frac{\mathrm{d} x \mathrm{~d} y}{x^{a q}}<\infty
$$

because $a q \leqslant(a+b) q<1$. In conclusion, $\mathrm{E}_{q}^{p}[h]<\infty$, as desired.

5.1. An extension. We just have constructed a monotone map $h: \overline{\mathbb{X}} \stackrel{\text { onto }}{\longrightarrow} \overline{\mathbb{Y}}$ of finite energy which equals the identity on the vertical sides of the rectangle $\overline{\mathbb{X}}=$ $[-1,1] \times[-2,2]$. However, restricted to the horizontal sides, it is not the identity; it takes the form

$$
h(x, 2)=\left(x|x|^{a}, 2\right) \text { and } h(x,-2)=\left(x|x|^{a},-2\right) \text { for }-1 \leqslant x \leqslant 1 .
$$

We shall still need a map that is equal to the identity on the entire boundary. For this reason we extend $h$ to a map $\widetilde{h}: \widetilde{\mathbb{X}} \stackrel{\text { onto }}{\longrightarrow} \widetilde{\mathbb{Y}}$, where $\widetilde{\mathbb{X}}=\widetilde{\mathbb{Y}}=[-1,1] \times[-3,3]$, by the rule

$$
\widetilde{h}(x, y)= \begin{cases}\left(x|x|^{a}(3-y)+x(y-2), y\right) & \text { when } 2 \leqslant y \leqslant 3 \\ h(x, y) & \text { when }-2 \leqslant y \leqslant 2, \\ \left(x|x|^{a}(3+y)-x(y+2), y\right) & \text { when }-3 \leqslant y \leqslant-2 .\end{cases}
$$

Clearly, $\widetilde{h}$ is monotone and equal to the identity on $\partial \widetilde{\mathbb{X}}$. Just as in the computation above, we see that $\mathrm{E}_{q}^{p}[\widetilde{h}]<\infty$. Proceeding further in this direction, we may extend $\widetilde{h}$ to the square $\mathbf{S} \stackrel{\text { def }}{=}[-4,4] \times[-4,4]$ by letting it be equal to the identity outside $\widetilde{\mathbb{X}}$. Let us record this in the following lemma.

Lemma 5.1. For every $p>2$ and $0<q<\frac{p}{p-2}$, there exists a noninjective monotone map $\Phi \in \mathscr{M}^{p}(\mathbf{S}, \mathbf{S})$ of finite $\mathbf{E}_{q}^{p}$-energy, which is the identity near the boundary of $\mathbf{S}$. Precisely, we have the following average energy:

$$
\mathbf{E}_{q}^{p}[\Phi]=\frac{1}{|\mathbf{S}|} \int_{\mathbf{S}}\left[|D \Phi(y)|^{p}+\frac{1}{[\operatorname{det} D \Phi(y)]^{q}}\right] \mathrm{d} y \stackrel{\text { def }}{=} \mathbf{E}<\infty,
$$

where $|\mathbf{S}|=16$ is the area of the square $\mathbf{S}=[-4,4] \times[-4,4]$. 
5.1.1. Rescaling. Formula (5.2) can be rescaled to an arbitrary square $Q \subset \mathbb{R}^{2}$ in place of $\mathbf{S}$. Let us discuss it in a somewhat greater context. Choose and fix a prototype energy integral over a square $\mathbf{S} \subset \mathbb{R}^{2}$ centered at the origin and of side length $L$ :

$$
\mathscr{E}[\Phi] \stackrel{\text { def }}{=} \frac{1}{|\mathbf{S}|} \int_{\mathbf{S}} \mathrm{E}(D \Phi(y)) \mathrm{d} y .
$$

This integral is assumed to exist for some adequate mappings $\Phi: \mathbf{S} \stackrel{\text { onto }}{\longrightarrow} \mathbf{S}, \Phi(0)=0$. Note that the stored-energy integrand depends solely on the deformation gradient $D \Phi$. Now take any square $Q$ centered at $a \in \mathbb{R}^{2}$ and of side length $\ell$. Then the mapping

$$
h_{Q}: Q \stackrel{\text { onto }}{\longrightarrow} Q \text {, defined by } h_{Q}(x) \stackrel{\text { def }}{=} a+\frac{\ell}{L} \Phi\left(\frac{L}{\ell}(x-a)\right),
$$

has the same average energy as $\Phi$ :

$$
\mathscr{E}\left[h_{Q}\right]=\frac{1}{|\mathbf{Q}|} \int_{\mathbf{Q}} \mathrm{E}\left(D h_{Q}(x)\right) \mathrm{d} x=\mathscr{E}[\Phi] .
$$

This is an obvious consequence of the chain rule $D h_{Q}(x)=D \Phi(y)$, where $y=$ $\frac{L}{\ell}(x-a)$ is a variable used as a substitution in the integral (5.3). For later use, it should be noted that if $\Phi$ is monotone, then so is $h_{Q}$. Also, if $\Phi$ is the identity map near $\partial \mathbf{S}$, then so is $h_{Q}$ near $\partial Q$.

\section{Cantor-type construction of Example 1.9.}

6.1. Construction of Cantor set. We shall work with closed squares whose sides are parallel to the standard coordinate axes of $\mathbb{R}^{2}$, but most of the definitions and formulas will be coordinate free.

6.1.1. Cornersquares. Suppose we are given a square $Q \subset \mathbb{R}^{2}$ and a parameter $0<\varepsilon<1$. Write it as $Q=I \times J$, where $I, J \subset \mathbb{R}$ are closed intervals of the same length $\ell=|I|=|J|$. These might be called, respectively, the horizontal and the vertical factors of $Q$. The notation $\varepsilon I$ and $\varepsilon J$ will stand for the intervals of the same centers but $\varepsilon$-times smaller in length, respectively. Cutting them out from $I$ and $J$ gives the decompositions

$$
I \backslash \varepsilon I=I_{-} \cup I_{+} \text {and } J \backslash \varepsilon J=J^{-} \cup J^{+}
$$

into the left and the right, as well as into the lower and the upper subintervals. Note that we suppressed the explicit dependence on $\varepsilon$ in the notation. This parameter will be determined later during our induction procedure. Now the Cartesian product consists of four subsquares:

$$
(I \backslash \varepsilon I) \times(J \backslash \varepsilon J)=Q_{+}^{+} \cup Q_{-}^{+} \cup Q_{-}^{-} \cup Q_{+}^{-} .
$$

Explicitly, we have the formulas

$$
Q_{+}^{+} \stackrel{\text { def }}{=} I_{+} \times J^{+}, Q_{-}^{+} \stackrel{\text { def }}{=} I_{-} \times J^{+}, Q_{-}^{-} \stackrel{\text { def }}{=} I_{-} \times J^{-}, Q_{+}^{-} \stackrel{\text { def }}{=} I_{+} \times J^{-} .
$$

Each of these subsquares touches exactly one corner of $Q$, which motivates our calling $Q_{+}^{+}, Q_{-}^{+}, Q_{-}^{-}, Q_{+}^{-}$the cornersquares of $Q$, more precisely, the first generation of cornersquares. We shall also spot the so-called centersquare of $Q$, defined by $\varepsilon Q=$ $\varepsilon I \times \varepsilon J$; see the left-hand side of Figure 2 . 


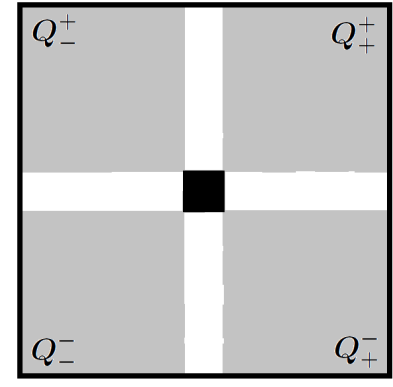

$Q_{\alpha_{1}}^{\beta_{1}} \in \mathcal{F}_{1}(\mathbb{Q})$

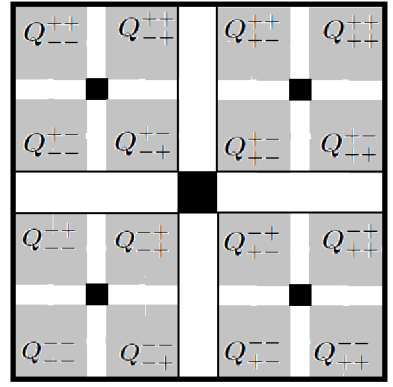

$Q_{\alpha_{1} \alpha_{2}}^{\beta_{1} \beta_{2}} \in \mathcal{F}_{2}(\mathbb{Q})$

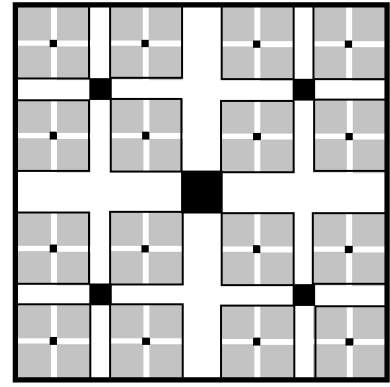

$Q_{\alpha_{1} \alpha_{2} \alpha_{3}}^{\beta_{1} \beta_{2} \beta_{3}} \in \mathcal{F}_{3}(\mathbb{Q})$

Cornersquares of the first, second and third generation

The branch set will develop in the black centersquares

FIG. 2. Cornersquares as building blocs for a Cantor-type construction.

6.1.2. Second generation of cornersquares. Choose another positive $\varepsilon$ parameter, say, $\varepsilon=\varepsilon_{2}$. Then every cornersquare of $Q$ gives rise to its own four cornersquares determined by this parameter; see the middle part of Figure 2. In this way we obtain 16 cornersquares of so-called second generation. According to our notation, these are

$$
\begin{array}{cccc}
Q_{++}^{++} & Q_{+-}^{++} & Q_{+-}^{+-} & Q_{++}^{+-} \\
Q_{-+}^{++} & Q_{--}^{++} & Q_{--}^{+-} & Q_{-+}^{+-} \\
Q_{-+}^{-+} & Q_{--}^{-+} & Q_{--}^{--} & Q_{-+}^{--} \\
Q_{++}^{-+} & Q_{+-}^{-+} & Q_{+-}^{--} & Q_{++}^{--}
\end{array}
$$

See also the third generation of 64 cornersquares in the right-hand side of Figure 2 .

6.1.3. The induction procedure. Fix a sequence of $\varepsilon$-parameters rapidly decreasing to 0 , say, $\left(\varepsilon_{1}, \varepsilon_{2}, \ldots\right)$ with $\varepsilon_{n}=4^{-n}$. We begin with the base $1 \times 1$ square $\mathbb{Q} \subset \mathbb{R}^{2}$ and the first $\varepsilon$ - parameter equal to $\varepsilon_{1}$. This gives us the first generation of four cornersquares $Q_{\alpha_{1}}^{\beta_{1}} \subset \mathbb{Q}$, where both indices run over the set $\{+,-\}$. We let $\mathcal{F}_{1}$ denote this family of cornersquares.

In the second step we take $\varepsilon_{2}$ as the $\varepsilon$-parameter and look at the cornersquares of every $Q_{\alpha_{1}}^{\beta_{1}}$. Denote them by $Q_{\alpha_{1} \alpha_{2}}^{\beta_{1} \beta_{2}} \subset Q_{\alpha_{1}}^{\beta_{1}}$, where $\alpha_{2}, \beta_{2} \in\{+,-\}$. They form the family $\mathcal{F}_{2}$ of second generation. More generally, given the family $\mathcal{F}_{n}$ of cornersquares $Q_{\alpha_{1} \alpha_{2} \ldots, \alpha_{n}}^{\beta_{1} \beta_{2}, \ldots, \beta_{n}} \subset Q_{\alpha_{1} \alpha_{2}, \ldots, \alpha_{n-1}}^{\beta_{1} \beta_{2}, \ldots, \beta_{n-1}} \in \mathcal{F}_{n-1}$, we take $\varepsilon_{n+1}$ as the $\varepsilon$-parameter and adopt to the family $\mathcal{F}_{n+1}$ the $\varepsilon$-cornersquares of $Q_{\alpha_{1} \alpha_{2}, \ldots, \alpha_{n}}^{\beta_{1} \beta_{2}, \ldots, \beta_{n}}$, namely,

$$
Q_{\alpha_{1} \alpha_{2}, \ldots, \alpha_{n}+}^{\beta_{1} \beta_{2}, \ldots, \beta_{n}+}, Q_{\alpha_{1} \alpha_{2}, \ldots, \alpha_{n}-}^{\beta_{1} \beta_{2}, \ldots, \beta_{n}+}, Q_{\alpha_{1} \alpha_{2}, \ldots, \alpha_{n}-}^{\beta_{1} \beta_{2}, \ldots, \beta_{n}-}, Q_{\alpha_{1} \alpha_{2}, \ldots, \alpha_{n}+}^{\beta_{1} \beta_{2}, \ldots, \beta_{n}-} \in \mathcal{F}_{n+1} .
$$

Thus, $\mathcal{F}_{n+1}$ consists of $4^{n+1}$ cornersquares denoted by $Q_{\alpha_{1} \alpha_{2}, \ldots, \alpha_{n} \alpha_{n+1}}^{\beta_{1} \beta_{2}, \ldots, \beta_{n} \beta n+1}$. This process continues indefinitely.

6.1.4. The size of squares in $\mathcal{F}_{n}$ and their total area. Let us compare the side length of squares in $\mathcal{F}_{n+1}$ with those in $\mathcal{F}_{n}$. Every member of $\mathcal{F}_{n+1}$ is a cornersquare of a $Q \in \mathcal{F}_{n}$ via the parameter $\varepsilon=\varepsilon_{n+1}$. Let $\ell$ denote the side length 
of $Q$. We remove from $Q$ its centersquare $\varepsilon Q$. Thus, each of the remaining four cornersquares has side length $\frac{1}{2}(1-\epsilon) \ell$. For $n=1$, this equals $\frac{1}{2}\left(1-\epsilon_{1}\right)$. Hence, by induction, the side length of squares in $\mathcal{F}_{n}$ equals $\frac{1}{2^{n}}\left(1-\varepsilon_{1}\right)\left(1-\varepsilon_{2}\right) \cdots\left(1-\varepsilon_{n}\right)<\frac{1}{2^{n}}$. We have $4^{n}$ such squares. This sums up to the total area of the union

$$
\left|\bigcup \mathcal{F}_{n}\right|=\left(1-\varepsilon_{1}\right)^{2}\left(1-\varepsilon_{2}\right)^{2} \cdots\left(1-\varepsilon_{n}\right)^{2} .
$$

6.1.5. The Cantor set. We have a decreasing sequence of compact sets $\bigcup \mathcal{F}_{1} \supsetneq$ $\cup \mathcal{F}_{2} \supsetneq, \ldots, \supsetneq \cup \mathcal{F}_{n}, \ldots$. Cantor's theorem tells us that their intersection is not empty:

$$
\mathcal{C} \stackrel{\text { def }}{=} \bigcap_{n-1}^{\infty}\left(\bigcup \mathcal{F}_{n}\right) \neq \emptyset
$$

The measure of this Cantor set is positive:

$$
|\mathcal{C}|=\lim _{n \rightarrow \infty}\left|\bigcup \mathcal{F}_{n}\right|=\prod_{k=1}^{\infty}\left(1-\varepsilon_{k}\right)^{2}>0 .
$$

The latter inequality is a consequence of $\sum_{k=1}^{\infty} \varepsilon_{k}<\infty$. Every point in $\mathcal{C}$ is obtained as the intersection of exactly one decreasing sequence of the form

$$
Q_{\alpha_{1}}^{\beta_{1}} \supsetneq Q_{\alpha_{1} \alpha_{2}}^{\beta_{1} \beta_{2}} \supsetneq \cdots \supsetneq Q_{\alpha_{1} \alpha_{2}, \ldots, \alpha_{n}}^{\beta_{1} \beta_{2}, \ldots, \beta_{n}} \ldots
$$

An obvious consequence of this is the following.

Lemma 6.1. Every open set that intersects $\mathcal{C}$ contains a square, say, $Q \in \mathcal{F}_{n}$, for sufficiently large $n$, which, in turn, contains its centersquare $\varepsilon_{n} Q \subset Q$.

The idea behind this lemma is a monotone mapping $h: \mathbb{Q} \stackrel{\text { onto }}{\longrightarrow} \mathbb{Q}$ whose branch set will materialize in the centersquares.

6.2. A monotone map $h: \mathbb{Q} \stackrel{\text { onto }}{\longrightarrow} \mathbb{Q}$. We let $\mathscr{G}$ denote the family of centersquares of all generations. From now on, the need will not arise for the explicit dependence on multi-indices in the notation of centersquares. For every $Q \in \mathscr{G}$, we have a monotone map $h_{Q}: Q \stackrel{\text { onto }}{\longrightarrow} Q$ defined by Formula (5.4) with $\Phi$ given in Lemma 5.1. Thus, the average $\mathrm{E}_{q}^{p}$-energy of $h_{Q}$ does not depend on $Q$ and equals E. In particular,

$$
\int_{Q}\left|D h_{Q}(x)\right|^{p} \mathrm{~d} x<\int_{Q}\left(\left|D h_{Q}(x)\right|^{p}+\left[J_{h_{Q}}(x)\right]^{-q}\right) \mathrm{d} x=|Q| \mathbf{E} .
$$

Recall that $h_{Q}$ equals the identity map near $\partial Q$. Now we can define the map $h \in \mathscr{M}^{p}(\overline{\mathbb{Q}}, \overline{\mathbb{Q}})$ that is hunted by Example 1.9.

Definition 6.2. We define the map $h: \mathbb{Q} \stackrel{\text { onto }}{\longrightarrow} \mathbb{Q}$ by setting

$$
h(x)= \begin{cases}h_{Q}(x) & \text { whenever } x \in Q \in \mathscr{G}, \\ x & \text { otherwise }\end{cases}
$$

Let us subtract the identity map:

$$
h(x)-x= \begin{cases}h_{Q}(x)-x \stackrel{\text { def }}{=} f_{Q}(x) & \text { whenever } x \in Q \in \mathscr{G}, \\ 0 & \text { otherwise }\end{cases}
$$

Copyright $@$ by SIAM. Unauthorized reproduction of this article is prohibited. 
One advantage of using this is that $f_{Q} \in \mathscr{W}_{0}^{1, p}(Q)$. Actually, $f_{Q}$ vanishes near $\partial Q$. We have the infinite series

$$
h(x)-x=\sum_{\mathbb{Q} \in \mathscr{G}} f_{Q}(x), \quad \text { in which } \sum_{\mathbb{Q} \in \mathscr{G}} \int_{Q}\left|D f_{Q}\right|^{p}<\infty .
$$

This latter inequality is due to the estimate (6.2). Now comes a general fact (rather folklore) about Sobolev functions.

Lemma 6.3. Let $\Omega \subset \mathbb{R}^{n}$ be a bounded domain and $\Omega_{i} \subset \Omega, i=1,2, \ldots$, disjoint open subsets. Suppose we are given functions $f_{i} \in \mathscr{W}_{0}^{1, p}\left(\Omega_{i}\right)$ such that $\sum_{i=1}^{\infty} \int_{\Omega_{i}}\left|D f_{i}(x)\right|^{p} \mathrm{~d} x<\infty$. Then the function

$$
f(x)= \begin{cases}f_{i}(x) & \text { whenever } x \in \Omega_{i}, \\ 0 & \text { otherwise }\end{cases}
$$

lies in the space $\mathscr{W}_{0}^{1, p}(\Omega)$.

We conclude that $h \in \mathscr{W}^{1, p}(\mathbb{Q}, \mathbb{Q})$ with $p>2$ and, as such, is continuous on $\overline{\mathbb{Q}}$. As regards monotonicity, for each square (continuum) $Q \in \mathscr{G}$, the mapping $h: Q \stackrel{\text { onto }}{\longrightarrow} Q$ is monotone, and $h$ is the identity outside those continua. This is enough to conclude that $h: \overline{\mathbb{Q}} \stackrel{\text { onto }}{\longrightarrow} \overline{\mathbb{Q}}$ is monotone. We leave the details to the reader.

Finally, every point of the Cantor set $\mathcal{C}$ belongs to the branch set of $h$. Indeed, by Lemma 6.1, any neighborhood of this point contains a square $Q \in \mathscr{G}$ in which $h=h_{Q}$ fails to be injective. Thus, the branch set $\mathcal{B}_{h}$ contains the Cantor set $\mathcal{C}$ and, therefore, has positive measure. On the other hand, by the very definition, $h(x) \equiv x$ on $\mathcal{C}$. Therefore, $h\left(\mathcal{B}_{h}\right)$ also contains $\mathcal{C}$, so $h\left(\mathcal{B}_{h}\right)$ has positive measure as well.

Remark 6.4. The branch set $\mathcal{B}_{h}$ consists precisely of the Cantor set $\mathcal{C}$ and vertical segments in the centersquares $Q \in \mathscr{G}$, which are squeezed to the centers. This makes it clear that $h\left(\mathcal{B}_{h}\right)=\mathcal{C}$. It should be noted that the branch set $\mathcal{B}_{h}$ can have nearly full measure. This follows from the formula (6.1) by letting $\varepsilon_{k}>0$ arbitrarily small.

The proof of Example 1.9 is complete.

6.3. Greater generality. We are now in a position to appreciate a more general approach to the construction presented above. Let us begin with an arbitrary bounded discrete set $\mathbf{G}$ of points in $\mathbb{R}^{2}$ whose limit set, denoted by $\mathbf{C}$, has positive area; see Figure 3. Clearly, $\mathbf{G}$ is necessarily countable. Moreover, $\mathbf{C} \cup \mathbf{G}$ is a compact subset of a bounded domain $\Omega \subset \mathbb{R}^{2}$. Given a point in $\mathbf{G}$, we may (and do) choose a square $Q \subset \Omega \backslash \mathbf{C}$ centered at this point and small enough so that the family of all such squares, denoted as before by $\mathscr{G}$, is disjoint. Analogously to Lemma 6.1 , every open set that intersects $\mathbf{C}$ contains a square in $\mathscr{G}$.

To every $Q \in \mathscr{G}$ there corresponds a monotone map $h_{Q}: Q \stackrel{\text { onto }}{\longrightarrow} Q$ equal to the identity near $\partial Q$. Recall the inequality (6.2):

$$
\int_{Q}\left|D h_{Q}(x)\right|^{p} \mathrm{~d} x<\int_{Q}\left(\left|D h_{Q}(x)\right|^{p}+\left[J_{h_{Q}}(x)\right]^{-q}\right) \mathrm{d} x=|Q| \mathbf{E} .
$$

This yields

$$
\sum_{Q \in \mathscr{G}} \int_{Q}\left|D h_{Q}(x)\right|^{p} \mathrm{~d} x<\sum_{Q \in \mathscr{G}} \mathrm{E}_{q}^{p}\left[h_{Q}\right]=\mathbf{E} \sum_{Q \in \mathscr{G}}|Q|<\mathbf{E}|\Omega|<\infty .
$$

Copyright $@$ by SIAM. Unauthorized reproduction of this article is prohibited. 


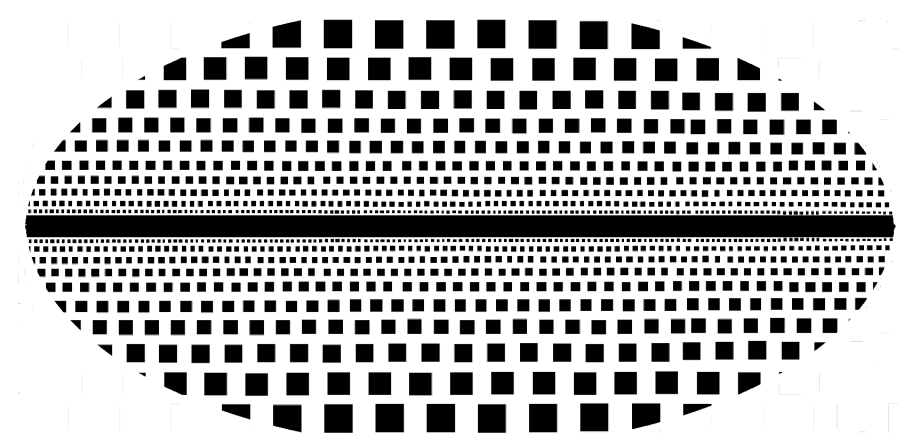

FIG. 3. Squares approaching the limit set.

It is precisely this property that one needs to infer $g \in \mathscr{W}^{1, p}(\Omega) \cap \mathscr{C}(\bar{\Omega})$. Exactly the same way as in Formula (6.3), we define a map $g: \Omega \stackrel{\text { onto }}{\longrightarrow} \Omega$ by the rule

$$
g(x)= \begin{cases}h_{Q}(x) & \text { whenever } x \in Q \in \mathscr{G} \\ x & \text { otherwise }\end{cases}
$$

Then we conclude in much the same way that $g \in \mathscr{M}^{p}(\bar{\Omega}, \bar{\Omega}), \mathrm{E}_{q}^{p}[g]<\infty,\left|\mathcal{B}_{g}\right|>0$, and $\left|g\left(\mathcal{B}_{g}\right)\right|>0$.

7. Proof of Theorem 1.2. Let $\mathbb{X}$ and $\mathbb{Y}$ be $\ell$-connected bounded Lipschitz domains in $\mathbb{R}^{2}$. Consider a family $\mathcal{F}$ of Sobolev orientation-preserving monotone mappings $h: \overline{\mathbb{X}} \stackrel{\text { onto }}{\longrightarrow} \overline{\mathbb{Y}}$ such that

$$
\mathrm{E}_{q}^{p}[h]=\int_{\mathbb{X}}\left(|D h|^{p}+\frac{1}{J_{h}^{q}}\right) \leqslant E
$$

for all $h \in \mathcal{F}$. Here $\mathbb{X}, \mathbb{Y}, p \geqslant 2, q>0$, and $E<\infty$ are fixed.

Lemma 7.1. The family $\mathcal{F}$ is equicontinuous. Precisely, there is a constant $C$ such that

$$
\left|h\left(x_{1}\right)-h\left(x_{2}\right)\right|^{2} \leqslant \frac{C \cdot E}{\log \left(1+\frac{C}{\left|x_{1}-x_{2}\right|}\right)}
$$

for all $h \in \mathcal{F}$ and distinct points $x_{1}, x_{2} \in \overline{\mathbb{X}}$.

Proof. Since $J_{h} \geqslant 0$ almost everywhere in $\mathbb{X}$, we have $h \in \mathscr{M}^{p}(\overline{\mathbb{X}}, \overline{\mathbb{Y}})$. Now, for proving (7.2) we may assume (equivalently) that $h \in \mathscr{H}^{p}(\mathbb{X}, \mathbb{Y})$ with $\mathrm{E}_{q}^{p}[h]<\infty$, thanks to Theorem 1.1. If $\mathbb{X}$ is multiple connected $\ell \geqslant 2$, then the modulus of continuity estimate (7.2) simply follows from the fact that the Dirichlet energy of $h$ is uniformly bounded by the value of neohookean energy $E$; see Lemma 2.2. Therefore, it suffices to consider the case of simply connected domains and $p=2$. It is worth recalling that if $\ell=1$, then $\int_{\mathbb{X}}|D h|^{2} \leqslant E$ is not enough to imply (7.2); see Remark 2.3.

Let $\ell=1$ and $p=2$. We may assume without loss of generality that $\mathbb{X}=\mathbb{D}=\mathbb{Y}$. Indeed, for any bounded Lipschitz domain $\mathbb{Y}$, there exists a global bi-Lipschitz change of variables $\Phi: \mathbb{C} \rightarrow \mathbb{C}$ for which $\Phi(\mathbb{Y})$ is the unit disk. Since the finiteness of the $\mathrm{E}_{q}^{2}$-energy is preserved under a bi-Lipschitz change of variables in both the target $\mathbb{Y}$ 
and the domain $\mathbb{X}$, we assume that $\mathbb{X}$ and $\mathbb{Y}$ are unit disks. Choose and fix any disk $B=B\left(x_{\circ}, \delta\right) \Subset \mathbb{X}$. We have, for every $h \in \mathcal{F}$,

$$
\begin{aligned}
|B| & =\int_{B} J_{h}^{\frac{q}{q+1}} \cdot \frac{1}{J_{h}^{\frac{q}{q+1}}} \\
& \leqslant\left(\int_{B} J_{h}\right)^{\frac{q}{q+1}}\left(\int_{B} \frac{1}{J_{h}^{q}}\right)^{\frac{1}{q+1}} \leqslant \mid h(B)^{\frac{q}{q+1}} \cdot E^{\frac{1}{q+1}} .
\end{aligned}
$$

Hence,

$$
|h(B)| \geqslant|B|^{\frac{q+1}{q}} E^{-\frac{1}{q}} \quad \text { constant independent of } h \in \mathcal{F} .
$$

Choose and fix $\varepsilon>0$ such that the annulus

$$
\Delta_{\varepsilon}=\{y \in \mathbb{Y}: \operatorname{dist}(y, \partial \mathbb{Y}) \leqslant \varepsilon\}
$$

has measure smaller than $|B|^{\frac{q+1}{q}} E^{-\frac{1}{q}}$. Thus, $h(B) \not \subset \Delta_{\varepsilon}$, and therefore there is a point $a \in B \Subset \mathbb{X}$ such that $|h(a)|<1-\varepsilon$. In other words, for every $h \in \mathcal{F}$ we can find a point $a \in \mathbb{X}$, with $|a| \leqslant 1-\delta$, and $b=h(a) \in \mathbb{Y}$, with $|b|<1-\varepsilon$. Now consider conformal mappings $\varphi: \overline{\mathbb{X}} \stackrel{\text { onto }}{\longrightarrow} \overline{\mathbb{X}}, \varphi(a)=0$, and $\psi: \overline{\mathbb{Y}} \rightarrow \overline{\mathbb{Y}}, \psi(b)=0$. Both mappings are bi-Lipschitz with bi-Lipschitz constants independent of $a$ and $b$. Thus, the energy $\psi \circ h \circ \varphi: \mathbb{X} \stackrel{\text { onto }}{\longrightarrow} \mathbb{Y}$ is controlled from above by that of $h$ uniformly in $\mathcal{F}$. Therefore, we may (and do) assume that $h(0)=0$. This leads us to the case of a homeomorphism $h: \mathbb{D} \backslash\{0\} \stackrel{\text { onto }}{\longrightarrow} \mathbb{D} \backslash\{0\}$, that is, between doubly connected domains. Finally, the inequality (7.2) follows from Lemma 2.2, completing the proof of Lemma 7.1.

Proof of Theorem 1.2. We apply the direct method in the calculus of variations. For that we take a minimizing sequence $h_{k} \in \mathscr{M}^{p}(\overline{\mathbb{X}}, \overline{\mathbb{Y}})$ of the neohookean energy $\mathrm{E}_{q}^{p}$ which converges weakly to $h_{\circ}$ in $\mathscr{W}^{1, p}(\mathbb{X}, \mathbb{C})$. Note that here we also used our standing assumption that the class of admissible homeomorphisms is nonempty. Therefore, by Lemma 2.7 we have

$$
\mathrm{E}_{q}^{p}\left[h_{\circ}\right] \leqslant \liminf _{k \rightarrow \infty} \mathrm{E}_{q}^{p}\left[h_{k}\right]=\inf _{h \in \mathscr{M}^{p}(\overline{\mathbb{X}}, \overline{\mathbb{Y}})} \mathrm{E}_{q}^{p}[h] .
$$

Since $\mathrm{E}_{q}^{p}\left[h_{k}\right] \leqslant E<\infty$ for every $k \in \mathbb{N}$ and $h_{k} \rightarrow h_{\circ}$ weakly in $\mathscr{W}^{1, p}(\mathbb{X}, \mathbb{C})$, applying Lemma 7.1 we see that the sequence $h_{k}$ also converges uniformly to $h_{\circ}$ in $\overline{\mathbb{X}}$. Now the mapping $h_{\circ}$, being a uniform limit of monotone mappings $h_{k}: \overline{\mathbb{X}} \stackrel{\text { onto }}{\longrightarrow} \overline{\mathbb{Y}}$, is a monotone map from $\overline{\mathbb{X}}$ onto $\overline{\mathbb{Y}}$. Therefore, $h_{\circ} \in \mathscr{M}^{p}(\overline{\mathbb{X}}, \overline{\mathbb{Y}})$. Combining this with (7.3) we have

$$
\mathrm{E}_{q}^{p}\left[h_{\circ}\right] \leqslant \inf _{h \in \mathscr{M}^{p}(\overline{\mathbb{X}}, \overline{\mathbb{Y}})} \mathrm{E}_{q}^{p}[h] \leqslant \mathrm{E}_{q}^{p}\left[h_{\circ}\right],
$$

finishing the proof of Theorem 1.2.

\section{Proof of Theorem 1.5.}

Proof. First we are going to estimate the distortion function

$$
1 \leqslant \mathscr{K}_{h}(x) \stackrel{\text { def }}{=} \frac{|D h(x)|^{2}}{2 J_{h}(x)}
$$

by using Young's inequality:

$$
A B C \leqslant \alpha A^{\frac{1}{\alpha}}+\beta B^{\frac{1}{\beta}}+\gamma C^{\frac{1}{\gamma}}, \quad A, B, C \geqslant 0 ; \alpha, \beta, \gamma \geqslant 0, \alpha+\beta+\gamma=1,
$$

Copyright (c) by SIAM. Unauthorized reproduction of this article is prohibited. 
where we add here to the convention that $\gamma C^{\frac{1}{\gamma}}=0$ for $\gamma=0$. The pointwise estimate of $\mathscr{K}_{h}$ by means of the energy integrand reads as, when $p>2$,

$$
\begin{aligned}
2 \mathscr{K}_{h}(x) & \leqslant \frac{2}{p}|D h|^{p}+\frac{1}{q} \frac{1}{J_{h}^{q}}+\left(1-\frac{2}{p}-\frac{1}{q}\right) \cdot 1 \\
& \leqslant \frac{2}{p}|D h|^{p}+\frac{1}{q} \frac{1}{J_{h}^{q}}+\left(1-\frac{2}{p}-\frac{1}{q}\right) \mathscr{K}_{h} .
\end{aligned}
$$

Hence,

$$
\mathscr{K}_{h} \leqslant|D h|^{p}+\frac{1}{J_{h}^{q}}=\mathrm{E}(|D h|, \operatorname{det} D h) .
$$

Integrating over $\Omega$ we obtain

$$
\left\|\mathscr{K}_{h}\right\|_{\mathscr{L}^{1}(\Omega)}=\int_{\Omega} \mathscr{K}_{h}(x) \mathrm{d} x<\infty .
$$

For Remark 1.6 concerning the case $p=2$ and $q=\infty$, we argue as follows:

$$
\mathscr{K}_{h}=\frac{|D h|^{2}}{2 J_{h}} \leqslant \frac{C}{2}|D h|^{2} .
$$

Hence,

$$
\int_{\mathbb{X}} \mathscr{K}_{h}<\frac{C}{2} \int_{\mathbb{X}}|D h|^{2}<\infty
$$

In either case $p>2$ or $p=2$, we see that the map $h \in \mathscr{W}^{1,2}\left(\Omega, \mathbb{R}^{2}\right)$ has integrable distortion. It is known [27] that such mappings $h: \Omega \rightarrow \mathbb{R}^{2}$ are discrete and open. In particular, $h(\Omega)$, being an open subset of $\overline{\mathbb{Y}}$, is contained in $\mathbb{Y}$. Next, we show that $h: \Omega \stackrel{\text { onto }}{\longrightarrow} h(\Omega)$ is injective. To this effect, suppose, to the contrary, that $h\left(x_{1}\right)=$ $h\left(x_{2}\right) \stackrel{\text { def }}{=} y_{\circ} \in h(\Omega)$ for some points $x_{1} \neq x_{2}$ in $\Omega$. The preimage $h^{-1}\left(y_{\circ}\right)$ under the map $h: \overline{\mathbb{X}} \stackrel{\text { onto }}{\longrightarrow} \overline{\mathbb{Y}}$ is a continuum in $\overline{\mathbb{X}}$ which contains $x_{1}, x_{2} \in \Omega$. This contradicts discreteness of $h: \Omega \rightarrow \mathbb{R}^{2}$.

Since $\Omega \Subset \mathbb{X}$ is arbitrary, it follows that $h: \mathbb{X} \stackrel{\text { onto }}{\longrightarrow} h(\mathbb{X})$ is a homeomorphism as well. Finally, it remains to show that $h(\mathbb{X})=\mathbb{Y}$. Certainly, $h(\mathbb{X})$, being an open subset of $\overline{\mathbb{Y}}$, is contained in $\mathbb{Y}$.

Suppose there is $y_{\circ} \in \mathbb{Y} \backslash h(\mathbb{X})$. But $y_{\circ} \in \overline{\mathbb{Y}}=h(\overline{\mathbb{X}})$, so $y_{\circ}=h\left(x_{\circ}\right)$ for some $x_{\circ} \in \overline{\mathbb{X}}$. On the other hand, the map $h: \overline{\mathbb{X}} \stackrel{\text { onto }}{\longrightarrow} \overline{\mathbb{Y}}$, being monotone, takes $\partial \mathbb{X}$ onto $\partial \mathbb{Y}$. This means that $x_{\circ} \notin \partial \mathbb{X}$ because $h\left(x_{\circ}\right)=y_{\circ} \notin \partial \mathbb{Y}$.

In conclusion,

$$
h(\mathbb{X})=\mathbb{Y} .
$$

\section{Proof of Theorem 1.10 .}

Proof. First, note that $s>p$ and $|D h|^{s} \in \mathscr{L}_{\text {loc }}^{1}(\mathbb{X})$. The idea of the proof is to infer from (1.7) that

$$
\frac{1}{J_{h}} \in \mathscr{L}_{\mathrm{loc}}^{\frac{s q}{p}}(\mathbb{X}) .
$$

For this purpose, consider the functions

$$
\begin{gathered}
\Phi=\left(1-\frac{p}{2}\right)|D h|^{p}+\frac{1+q}{J_{h}^{q}} \in \mathscr{L}_{\text {loc }}^{1}(\mathbb{X}), \\
\Psi=2 p|D h|^{p-2} \overline{h_{z}} h_{\bar{z}} \in \mathscr{L}_{\mathrm{loc}}^{r}(\mathbb{X}, \mathbb{C}), \quad \text { where } r=\frac{s}{p}>1 .
\end{gathered}
$$

Copyright (c) by SIAM. Unauthorized reproduction of this article is prohibited. 
Equation (1.7) reads as

$$
\Phi_{\bar{z}}=\Psi_{z} \quad \text { in the sense of distributions. }
$$

We first observe the following.

Lemma 9.1. For every subdomain $\Omega \Subset \mathbb{X}$ compactly contained in $\mathbb{X}$, it holds that

$$
\Phi \in \mathscr{L}^{r}(\Omega) .
$$

Proof of Lemma 9.1. Choose and fix a function $\lambda \in C_{0}^{\infty}(\mathbb{X})$ which equals 1 in a neighborhood of $\bar{\Omega} \subset \mathbb{X}$. Then consider the expression defined in the entire complex plane by the rule

$$
\lambda \Phi-S \lambda \Psi,
$$

where $S: \mathscr{L}^{r}(\mathbb{C}) \rightarrow \mathscr{L}^{r}(\mathbb{C})$ is the Beurling-Ahlfors transform

$$
(S f)(z)=-\frac{1}{\pi} \int_{\mathbb{C}} \int \frac{f(\xi) \mathrm{d} \xi}{(z-\xi)^{2}} \text { for } f \in \mathscr{L}^{r}(\mathbb{C}) .
$$

The following identity is characteristic of Beurling-Ahlfors transform:

$$
\frac{\partial}{\partial \bar{z}}(S f)=\frac{\partial}{\partial z} f \text { for } f \in \mathscr{L}^{r}(\mathbb{C}) .
$$

The complex derivatives are understood in the sense of distributions. We may (and do) apply this identity to $f=\lambda \Psi$. Differentiating the expression (9.5) with respect to $\bar{z}$ yields

$$
\begin{aligned}
\frac{\partial}{\partial \bar{z}}[\lambda \Phi-S \lambda \Psi] & =\frac{\partial}{\partial \bar{z}}[\lambda \Phi]-\frac{\partial}{\partial z}[\lambda \Psi] \\
& =\lambda_{\bar{z}} \Phi+\lambda \Phi_{\bar{z}}-\lambda_{z} \Psi-\lambda \Psi_{z} \\
& =\lambda_{\bar{z}} \Phi-\lambda_{z} \Psi=0 \text { in a neighborhood of } \bar{\Omega} .
\end{aligned}
$$

Here we used (9.4) and the fact the $\lambda \equiv 1$ in a neighborhood of $\bar{\Omega}$. Thus, by Weyl's lemma, the function

$$
H=\lambda \Phi-S \lambda \Psi
$$

is holomorphic in a neighborhood of $\bar{\Omega} \subset \mathbb{X}$, so $H \in \mathscr{L}^{r}(\Omega)$. In this neighborhood we express $H$ as

$$
H(z)=\Phi(z)+\frac{1}{\pi} \int_{\mathbb{C}} \int \frac{\lambda(\xi) \Psi(\xi) \mathrm{d} \xi}{(z-\xi)^{2}} .
$$

The latter integral, being the Beurling-Ahlfors transform of $\lambda \Psi \in \mathscr{L}^{r}(\mathbb{C})$, represents a function in $\mathscr{L}^{r}(\mathbb{C})$. In conclusion, $\Phi \in \mathscr{L}^{r}(\Omega)$.

Now it follows by (9.2) that $J_{h}^{-q} \in \mathscr{L}_{\text {loc }}^{r}(\Omega)$, as claimed in (9.1). Combining this with the assumption $|D h|^{s} \in \mathscr{L}_{\text {loc }}^{1}(\mathbb{X})$, we have

$$
\int_{\Omega}\left(|D h|^{s}+J_{h}^{-\frac{s q}{p}}\right)<\infty
$$

where $\Omega \Subset \mathbb{X}, s>2$, and $\frac{s q}{p} \geqslant \frac{s}{s-2}$. We are now in a position to apply Theorem 1.5 for $s$ in place of $p$ and $\frac{s q}{p}$ in place of $q$. Therefore,

$$
h: \mathbb{X} \stackrel{\text { onto }}{\longrightarrow} \mathbb{Y}
$$

is a homeomorphism. 
Acknowledgments. We thank the referees for careful reading of the manuscript and helpful comments.

\section{REFERENCES}

[1] S. S. Antman, Nonlinear Problems of Elasticity. Applied Mathematical Sciences 107, SpringerVerlag, New York, 1995.

[2] K. Astala, T. Iwaniec, And G. Martin, Deformations of annuli with smallest mean distortion, Arch. Ration. Mech. Anal., 195 (2010), pp. 899-921.

[3] K. Astala, T. Iwaniec, and G. Martin, Elliptic Partial Differential Equations and Quasiconformal Mappings in the Plane, Princeton University Press, Princeton, NJ, 2009.

[4] J. M. BALL, Convexity conditions and existence theorems in nonlinear elasticity, Arch. Ration. Mech. Anal., 63 (1976/77), pp. 337-403.

[5] J. M. BALL, Global invertibility of Sobolev functions and the interpenetration of matter, Proc. Roy. Soc. Edinb. Sect. A, 88 (1981), pp. 315-328.

[6] J. M. BALL, Existence of solutions in finite elasticity, in Proceedings of the IUTAM Symposium on Finite Elasticity, Martinus Nijhoff, Dordrecht, the Netherlands, 1981, pp. 1-12.

[7] J. M. BALL, Discontinuous equilibrium solutions and cavitation in nonlinear elasticity, Philos. Trans. Roy. Soc. A, 306 (1982), pp. 557-611.

[8] P. Bauman, D. Phillips, AND N. OWen, Maximum principles and a priori estimates for an incompressible material in nonlinear elasticity, Comm. Partial Differential Equations, 17 (1992), pp. 1185-1212.

[9] P. G. Ciarlet, Mathematical elasticity Vol. I. Three-dimensional elasticity, Studies in Mathematics and Its Applications 20, North-Holland, Amsterdam, 1988.

[10] P. G. Ciarlet And J. NEČAS, Injectivity and self-contact in nonlinear elasticity, Arch. Ration. Mech. Anal., 97 (1987), pp. 171-188.

[11] S. Conti And C. De Lellis, Some remarks on the theory of elasticity for compressible Neohookean materials, Ann. Sc. Norm. Super. Pisa Cl. Sci. (5), 2 (2003), pp. 521-549.

[12] J. Cristina, T. Iwaniec, L. V. Kovalev, and J. Onninen, The Hopf-Laplace equation: Harmonicity and regularity, Ann. Sc. Norm. Super. Pisa Cl. Sci. (5), 13 (2014), pp. 1145-1187.

[13] L. C. Evans, Quasiconvexity and partial regularity in the calculus of variations, Arch. Ration. Mech. Anal., 95 (1986), pp. 227-252.

[14] I. Fonseca And W. Gangbo, Local invertibility of Sobolev functions, SIAM J. Math. Anal., 26 (1995), pp. 280-304.

[15] P. HaJŁasz And J. MalÝ, Approximation in Sobolev spaces of nonlinear expressions involving the gradient, Ark. Mat., 40 (2002), pp. 245-274.

[16] D. Henao And R. Rodiac, On the existence of minimizers for the neo-Hookean energy in the axisymmetric setting, Discrete Contin. Dyn. Syst., 38 (2018), pp. 4509-4536.

[17] S. Hencl And P. Koskela, Lectures on mappings of finite distortion, Lecture Notes in Mathematics 2096, Springer, New York, 2014.

[18] T. Iwaniec and G. Martin, Geometric Function Theory and Non-Linear Analysis, Oxford Mathematical Monographs, Oxford University Press, Oxford, 2001.

[19] T. Iwaniec, P. Koskela, And J. Onninen, Mappings of finite distortion: Monotonicity and continuity, Invent. Math., 144 (2001), pp. 507-531.

[20] T. Iwaniec, L. V. Kovalev, And J. Onninen, Lipschitz regularity for inner-variational equations, Duke Math. J., 162 (2013), pp. 643-672.

[21] T. Iwaniec And J. OnNinen, Neohookean deformations of annuli, existence, uniqueness and radial symmetry, Math. Ann., 348 (2010), pp. 35-55.

[22] T. Iwaniec And J. Onninen, Deformations of finite conformal energy: Boundary behavior and limit theorems, Trans. Amer. Math. Soc. 363 (2011), pp. 5605-5648.

[23] T. Iwaniec And J. Onninen, $n$-Harmonic mappings between annuli, Mem. Amer. Math. Soc. $218(2012)$.

[24] T. Iwaniec And J. OnNinen, Mappings of least Dirichlet energy and their Hopf differentials, Arch. Ration. Mech. Anal., 209 (2013), pp. 401-453.

[25] T. Iwaniec And J. Onninen, Monotone Sobolev mappings of planar domains and surfaces, Arch. Ration. Mech. Anal., 219 (2016), pp. 159-181.

[26] T. Iwaniec And J. Onninen, Limits of Sobolev homeomorphisms, J. Eur. Math. Soc., 19 (2017), pp. $473-505$.

[27] T. Iwaniec And V. Šverák, On mappings with integrable dilatation, Proc. Amer. Math. Soc. 118 (1993), no. 1, pp. 181-188.

Copyright (c) by SIAM. Unauthorized reproduction of this article is prohibited. 
[28] A. Koski and J. Onninen, Radial symmetry of p-harmonic minimizers, Arch. Ration. Mech. Anal., 230 (2018), pp. 321-342.

[29] H. Lebesgue, Sur le probléme de Dirichlet, Rend. Circ. Palermo, 27 (1907), pp. 371-402.

[30] C. B. Morrey, The Topology of (Path) Surfaces, Amer. J. Math., 57 (1935), pp. 17-50.

[31] C. B. Morrey, Quasiconvexity and the semicontinuity of multiple integrals, Pacific J. Math., 2 (1952), pp. 25-53.

[32] S. MÜller, T. QI, AND B. S. Yan, On a new class of elastic deformations not allowing for cavitation, Ann. Inst. H. Poincaré Anal. Non Linéaire, 11 (1994), pp. 217-243.

[33] S. Müller And S. J. Spector, An existence theory for nonlinear elasticity that allows for cavitation, Arch. Ration. Mech. Anal., 131 (1995), pp. 1-66.

[34] Yu. G. Reshetnyak, Space Mappings with Bounded Distortion, American Mathematical Society, Providence, RI, 1989.

[35] J. Sivaloganathan And S. J. Spector, Necessary conditions for a minimum at a radial cavitating singularity in nonlinear elasticity, Ann. Inst. H. Poincaré Anal. Non Linéaire, 25 (2008), pp. 201-213.

[36] L. R. G. Treloar, The Physics of Rubber Elasticity, Oxford University Press, Oxford, 1975.

Copyright (c) by SIAM. Unauthorized reproduction of this article is prohibited. 